\title{
Photoémission de Csl induite par une impulsion laser intense femtoseconde
}

\author{
A. Belsky ${ }^{1,2}$, A. Vasil'ev', B. Yatsenko ${ }^{1}$, H. Bachau, ${ }^{2}$, P. Martin ${ }^{2}$, \\ G. Geoffroy ${ }^{3}$ et S. Guizard ${ }^{3}$ \\ ${ }^{1}$ Department of Optics and Spectroscopy, Moscow Lomonosov University, 11234 Moscow, \\ Russie \\ ${ }^{2}$ Centre Lasers Intenses et Applications, Université de Bordeaux l et CNRS, 33405 Talence, \\ France \\ ${ }^{3}$ Laboratoire des Solides Irradiés, CEA/DSM/DRECAM-CNRS, École Polytechnique, \\ 91128 Palaiseau cedex, France
}

\begin{abstract}
Résumé. Nous avons mesuré pour la première fois les spectres de photoélectrons émis par un cristal isolant à large bande interdite, Csl, avec une dynamique de $10^{6} \mathrm{coups} / \mathrm{s}$, excité par la source laser haute cadence du C.E.L.I.A ( 800 $\mathrm{nm}, 40 \mathrm{fs}, 1 \mathrm{kHz}, 1 \mathrm{TW}$ ). L'émission d'électrons jusqu'à des énergies de quelques dizaines d'électrons-volts a été observée pour des impulsions d'éclairement compris entre 0.5 et $3 \mathrm{TW} / \mathrm{cm}^{2}$, relativement faible donc par comparaison aux éclairements utilisés pour accélérer les électrons d'un atome aux mêmes énergies. Ces spectres contiennent tous, en particulier, deux bandes dans le domaine des basses énergies d'électrons $(<5 \mathrm{eV})$, également observées lors d'études précédentes. Les électrons les plus énergétiques forment un plateau intense légèrement structuré et limité par une coupure exponentielle. Pour des impulsions de $3 \mathrm{TW} / \mathrm{cm}^{2}$ cette coupure est située à $27 \mathrm{eV}$.

L'insuffisance du mécanisme électron-photon-phonon, considéré jusqu'à présent comme le principal processus d'échauffement des électrons dans les solides en interaction non destructrice avee un champ laser, nous a poussé à proposer un mécanisme alternatif. Ce modèle met en évidence les transitions directes multiphotoniques dans la bande de conduction du solide qui sont incontournables du fait de sa structure électronique multi-branches
\end{abstract}

\section{INTRODUCTION}

L'interaction d'impulsions laser intenses avec les atomes est activement étudiée depuis des années, une des principales applications étant la génération d'harmoniques d'ordres élevés dans le domaine de l'UVX. Par contre, les détails de cette interaction avec les solides, en particulier avec les cristaux diélectriques, sont beaucoup moins connus. Les principales études ont été centrées d'une part sur l'interaction avec des impulsions de grande puissance capables de créer un plasma à la surface du solide, d'autre part aux effets de l'absorption multiphotonique induite par des champs relativement faibles. C'est pourquoi notre intérêt porte sur l'étude des phénomènes fortement non-linéaires et non destructifs, générés lors de l'interaction d'impulsions laser de puissance intermédiaire avec les solides. L'étude de ces phénomènes pourrait conduire à la connaissance des mécanismes physiques de claquage optique et de génération de rayonnement UVX par les solides isolants.

\section{RESULTATS ET DISCUSSION}

Nous avons mesuré à l'aide de la source laser du CELIA [1] les spectres d'électrons émis par le cristal diélectrique CsI excités par des impulsions de $40 \mathrm{fs}$, d'énergie variant de 20 à $100 \mu \mathrm{J}$, à la longueur d'onde de $800 \mathrm{~nm}$, et focalisées sur la surface du cristal pour atteindre des éclairements compris entre 0.5 et $3 \mathrm{TW} / \mathrm{cm}^{2}$. Le cristal, installé dans la chambre expérimentale [2] sous une pression de $5 \times 10^{-9}$ torr, est chauffé à $200^{\circ} \mathrm{C}$ pour neutraliser la charge de surface. Le spectromètre de photoélectrons (CLAM 4, VG Microtech) est équipé de 9 channeltrons. A la cadence du laser de $1 \mathrm{kHz}$ on peut, en variant la tension d'alimentation des channeltrons, élargir la dynamique d'acquisition jusqu'à $10^{6} \mathrm{coups} / \mathrm{s}$, tout en conservant la linéarité du comptage des électrons.

Le résultat principal concerne l'observation de modifications importantes de la partie haute énergie des spectres de photoélectrons. L'augmentation de l'éclairement est accompagnée par l'apparition d'un plateau composé d'électrons d'énergie de quelques dizaines d'eV, limité par une coupure d'allure 
exponentielle (fig.1). Ainsi, pour une excitation de $3 \mathrm{TW} / \mathrm{cm}^{2}$ l'énergie cinétique maximale atteinte par les électrons est $\sim 27 \mathrm{eV}$. Le rendement en électrons dans la région du plateau est comparable à celui des électrons de basse énergie. Ce phénomène paraît universel puisque pour une excitation similaire, des électrons de 20-40 eV ont été observés dans des expériences sur $\mathrm{SiO}_{2}$ [2], $\mathrm{CeF}_{3}$ et $\mathrm{Al}_{2} \mathrm{O}_{3}$ [3].

Le spectre de photoélectrons pourrait être modifié par le phénomène de charge d'espace, du à l'échange d'énergie entre les électrons compris dans un «paquet» court et dense durant son parcours de l'échantillon vers le détecteur. La présence d'un potentiel d'extraction de plusieurs $\mathrm{kV}$ amplifie considérablement l'effet [4]. Nous estimons que, dans nos conditions expérimentales, l'influence d'un phénomène de charge d'espace sur les spectres est négligeable (inférieur à $1 \mathrm{eV}$ ). La signature d'un effet de charge d'espace est un élargissement symétrique et un décalage des spectres vers les hautes énergies cinétiques. Ceci est contraire à nos observations. En effet la structure située à $2.5 \mathrm{eV}$ reste stable en largeur et position, même pour les intensités les plus élevées. D'autre part nous utilisons un analyseur hémisphérique qui disperse les électrons suivant leur énergie cinétique, et le potentiel le plus élevé auquel sont soumis les électrons dans les lentilles de focalisation d'entrée est toujours inférieur à 40 volts.

On peut remarquer certaines similitudes entre nos spectres et ceux des électrons résultant de l'ionisation atomique dans un champ intense (spectres ATI). Cependant les plateaux dans les spectre ATI des atomes apparaissent pour des intensités environ deux ordres de grandeur supérieures à celles utilisées
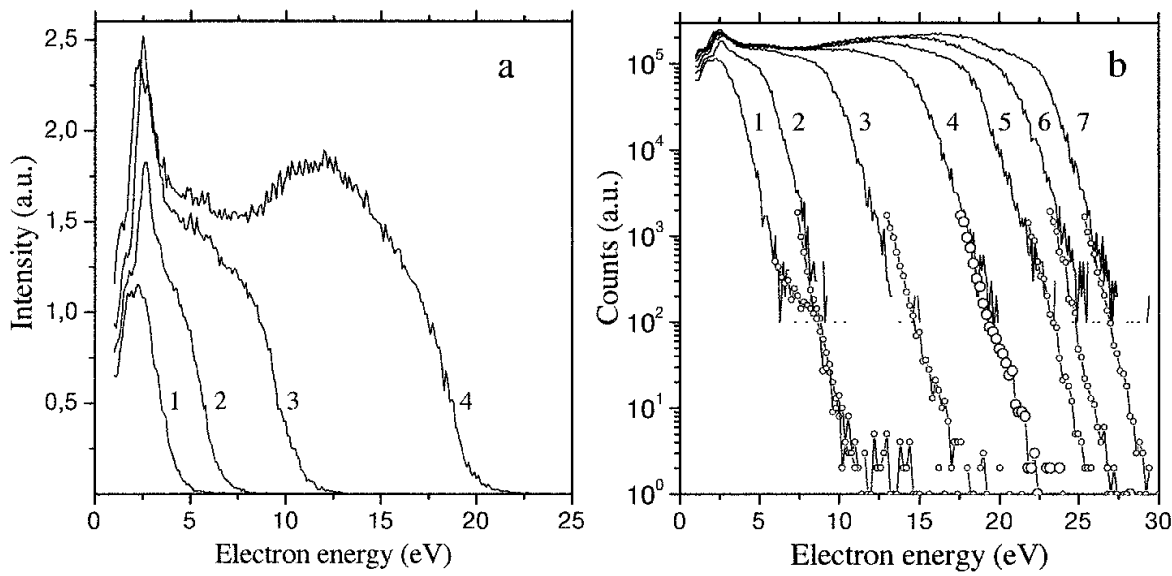

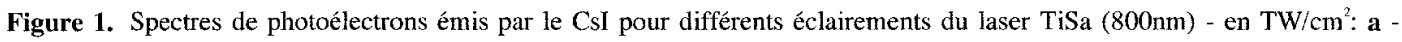
0.5 (courbe 1), 0.7(2), 1(3), 2(4); b - 0.5(1), 0.7(2), 1(3), 1.5(4), 2( 5), 2.5(6), 3(7).

lors de nos expériences. A $1 \mathrm{TW} / \mathrm{cm}^{2}$ l'énergie cinétique maximale acquise par un électron libre est largement inférieure aux énergies mesurées pour un solide diélectrique. Il paraît évidant que les électrons de haute énergie, avec le rendement relativement fort observé, sont dus au phénomène spécifique de l'interaction du champ intense avec le solide.

Les modèles de l'échauffement d'électrons dans un solide à large bande interdite par un champ intense font essentiellement appel à l'interaction electron-photon-phonon [5-8]. La partie de basse énergie des spectres mesurés (Fig.1) est similaire aux résultats obtenus auparavant pour $\mathrm{SiO}_{2}$ [8]. En accord avec ces études, la première bande (un épaulement vers $1 \mathrm{eV}$ ) peut être attribuée aux électrons directement excités de la bande de valence par transition multiphotonique (ou à par l'intermédiaire des états localisés dans la bande interdites) qui ne subissent pas d'échauffement. La largeur totale de cette bande peut être estimée inférieure à $1.5 \mathrm{eV}$, mais sa partie faible énergie est coupée par l'énergie du travail de sortie des électrons. Suivant ce modèle la deuxième bande $(2.5 \mathrm{eV})$ peut être attribuée aux électrons qui ont absorbé un photon supplémentaire par interaction électron-phonon-photon, la probabilité de telles transitions est contrôlée par le temps caractéristique d'interaction électron-phonon. L'échauffement des électrons jusqu'à des énergies de $30 \mathrm{eV}$ suppose un grand nombre d'actes successifs d'interaction électron-phonon-photons. Etant donnée la faible probabilité d'une telle succession d'évènements, le spectre devrait alors présenter une pente décroissante à partir $\mathrm{de} \sim 3 \mathrm{eV}$, ce qui est en contradiction avec nos résultats. Finalement, en 
tenant compte du temps caractéristique d'interaction électron-phonon dans le cristal de CsI $(\sim 10 \mathrm{fs})$ proche de la durée des impulsions laser (40 fs), nous concluons que le plateau dans le spectre de photoélectrons ne peut pas être attribué à l'échauffement par interaction electron-photon-phonon.

\section{PROPOSITION DE MODELE}

Le modèle de l'échauffement d'électrons dans les solides que nous proposons est basé sur un phénomène de transitions optiques directes entre les branches de la bande de conduction qui n'ont pas été prises en compte dans les modèles précédents bien qu'elles aient un caractère universel. Les transitions directes radiatives d'électrons dans la bande de conduction avec un rendement spectral de $10^{-4}-10^{-5}$ photon/électron/eV ont été observées dans les solides à large bande interdite sous excitation par rayonnements ionisants, capables de créer les électrons chauds [9]. Cette luminescence ultra-rapide intrabande, qui subit une forte concurrence avec la relaxation rapide non-radiative (interaction électronphonon), est observable seulement si les éléments de matrice de transition entre les branches de la bande de conduction sont comparables à ceux des transitions directe à partir de la bande de valence. Comme les derniers sont bien étudiés expérimentalement, nous pouvons estimer que la section efficace d'absorption d'un photon par un électron dans la bande de conduction est proche de $10^{-16}-10^{-17} \mathrm{~cm}^{2}$.

La probabilité $\left(\mathrm{W}_{2 I}\right)$ des transitions multiphotoniques directes entre les branche 1 et 2 dans la bande de conduction en fonction de champ intense optique $(F)$ peut être présentée sous la forme suivante [11]:

$$
\begin{aligned}
& W_{21}=\frac{2 \pi}{\hbar} \int \frac{d^{3} p}{(2 \pi \hbar)^{3}} \mid L_{21}(\vec{p})^{2} \sum_{n} \delta(\bar{\varepsilon}(\vec{p} ; F)-n \hbar \omega) \\
& \text { où } \bar{\varepsilon}(\vec{p} ; F)=\frac{1}{2 \pi} \int_{-\pi}^{\pi} \varepsilon\left(\vec{p}+\frac{e \vec{F}}{\omega} \sin x\right) d x \text { et } \varepsilon(\vec{p})=\varepsilon_{2}(\vec{p})-\varepsilon_{1}(\vec{p})
\end{aligned}
$$

sont respectivement l'énergie de transition moyennée par la phase du champ et l'écart énergétique entre les branches 1 et 2 pour une même valeur de la quasi-impulsion.

Le calcul direct de la probabilité $\mathrm{W}_{21}$ pour un électron dans la bande de conduction du cristal demande des procédures numériques complexes et lourdes, basées sur le calcul de la structure des bandes et des fonctions d'ondes des électrons. L'application, pour simplifier le calcul, de l'approximation de la masse effective, traditionnellement utilisée pour la description des transition multiphotoniques à partir de la bande de valence et dans la plupart des modèles d'échauffement des électrons dans un solide, est impossible. Cette approche est valable seulement pour des électrons de faible énergie (quelques $\mathrm{eV}$ ), inférieure à l'énergie cinétique d'un électron aux frontières de la zone Brillouin. La description d'électrons d'énergie plus importante exige les modèles, qui prennent en compte la périodicité du potentiel cristallin et la structure multi-branche de la bande de conduction. C'est, en particulier, l'approximation des électrons quasi-libres, qui, en sa forme standard, facilite le calcul mais ne permet pas d'éviter les techniques numériques.

Pour mettre en évidence le rôle des transition directes dans l'échauffement des électron dans un solide par un champ optique intense nous avons choisi l'approximation MPBB (multiple-parabolic-branch band) qui a été utilisée auparavant pour la description de la relaxation d'électrons chauds dans les diélectriques [10]. Dans l'approximation MPBB les élément de matrice de transition dans un champ optique faible sont remplacés par la valeur moyenne constante qu'on estime à partir de donnés extérieures (par exemple expérimentales). Cette simplification permet d'une part de garder l'information sur le potentiel cristallin, d'autre part d'utiliser seulement la partie périodique de la fonction d'onde de Bloch et une simple structure électronique multi-branche de la bande de conduction. Dans cette représentation, les courbes de dispersion d'énergie d'électrons dans la bande de conduction sont présentées comme une série de paraboles $\varepsilon_{i}(\vec{p})=\left(\vec{p}-\vec{p}_{i}\right)^{2} / 2 m$, où $\varepsilon_{i}$ est l'énergie d'un électron avec la quasi-impulsion $\vec{p}$ sur la branche $i, m$ sa masse et $\vec{p}_{i}$ un des vecteurs du réseau réciproque. Cette loi de dispersion néglige les gaps en limite de zone de Brillouin, dus au potentiel cristallin non-nul, mais il reste correct dans l'essentiel de la zone ou se produisent les transitions en considération. 
Les transitions directes entre les branches sont contrôlées par les lois de conservation de l'impulsion et de l'énergie. Pour cette raison les transitions directes entre les branches, avec l'absorption d'un photon dans un champ optique faible (Fig.2, flèches continus), ne sont possibles que si un électron a déjà acquis une énergie cinétique considérable. En présence d'un champ intense les transitions multiphotoniques entre les branches deviennent possibles (Fig.2, flèches en pointillé), elles commencent, pour la même paire de branches, à partir d'énergies électroniques plus faibles. L'interaction avec le champ intense $\vec{F}(t)=\vec{F} \cos (\omega t)$ induit des oscillations importantes de la quasi-impulsion d'un électron $\vec{p}(t)=\vec{p}+(e \vec{F} / \omega) \sin (\omega t)$; dans ce cas, l'énergie d'un électron d'impulsion $p$ sera caractérisée non pas par l'énergie fixe $\varepsilon_{i}$, mais par une énergie moyennée sur la période d'oscillation du champ (2) généralement différente de $\varepsilon_{i}$. Ce phénomène est similaire à celui connu pour les atomes. Notons, que pour la loi de dispersion que nous utilisons, le champ intense ne change

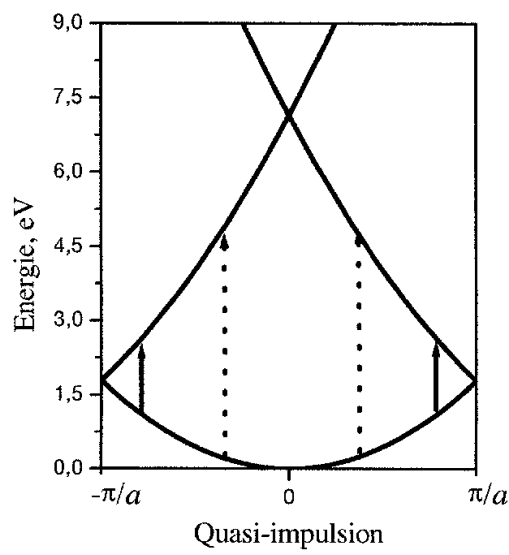

Figure 2. La structure électronique de la bande de conduction dans l'approximation MPBB; les transitions directes à un photon (ligne continue) et à trois photon (pointillé) pas l'énergie moyenne de transition, il induit le même déplacement d'énergie pour toutes les branches, ce déplacement est égal au potentiel pondéromoteur.

En présence d'un champ intense l'élément de matrice de transition de la première branche vers la deuxième a la forme suivante [11]:

$$
L_{21}(\vec{p})=\frac{1}{2 \pi} \oint_{C} V_{21}\left(\vec{p}+\frac{e \vec{F}}{\omega} u\right) \operatorname{Exp}\left\{\frac{i}{\hbar \omega} \int_{0}^{u} \varepsilon\left(\vec{p}+\frac{e \vec{F}}{\omega} v\right) \frac{d v}{\left(1-v^{2}\right)^{1 / 2}}\right\} d u
$$

où $C$ est le contour d'intégration par la phase du champ et $V_{21}(\vec{p})$ est l'élément de matrice de transition dans un champ faible. Pour les calcules ci-dessous nous supposons que $V_{21}(\vec{p})=V_{0} \sqrt{I}$ où $I$ est l'intensité de champ et $V_{0}$ est une constante que nous estimons à partir de données expérimentales pour la section efficace d'absorption d'un électron de la bande de conduction dans le champ optique faible. L'application de l'approximation MPBB nous à permis de réaliser le calcul analytique de l'intégrale dans la formule (3):

$$
\mid L_{21}(n, \vec{F})^{2}=\frac{V_{0}^{2} I}{B_{1}^{2}}\left(B_{1} J_{n-1}\left(B_{1}\right)-n J_{n}\left(B_{1}\right)\right)^{2}
$$

ou $J_{n}(x)$ sont les fonctions de Bessel et $B_{1}=\frac{1}{\hbar \omega} \frac{e \vec{F}\left(\vec{p}_{2}-\vec{p}_{1}\right)}{\omega m}$.

La solution est similaire au cas de la diffusion d'un électron dans un potentiel non-périodique local [12], elle est exprimée par des fonctions de Bessel. Mais le potentiel périodique, que nous utilisons, limite les variations de quasi-impulsion, par des valeurs proportionnelles au vecteur du réseau réciproque. Cette condition conduit à l'observation des oscillations dans les spectres de probabilité de transition multiphotonique dans un cristal (voir Fig.3). Les probabilités par femtoseconde de transitions directes à un, deux et trois photons sont calculées d'après les formules (1) et (4) en utilisant les paramètres de la structure cristalline de CsI. Le profil des oscillations et la position des minima dépendent également de l'orientation du champ optique par rapport aux axes cristallins (le calcul est fait ici pour un champ parallèle à la direction [111]). L'utilisation de la résolution angulaire devrait donc permettre une étude affinée de ce phénomène d'échauffement par transitions directes. Les même courbes sont monotones dans le cas atomique, comme en l'absence de la limitation induite par le potentiel périodique l'intégration dans toutes les directions lisse les oscillations.

Dans la gamme des éclairements $1-10 \mathrm{TW} / \mathrm{cm}^{2}$ on observe que la probabilité des transitions multiphotoniques devient comparable à celles à un seul photon. Notons que ces transitions peuvent avoir lieu à partir d'états électroniques situés sur la courbe de dispersion à une énergie plus basse que celle du seuil de la transition à un photon (Fig.2). Ainsi les transitions directes peuvent intervenir dès le début de 
l'échauffement, à partir des énergies apportées par l'ionisation de la bande de valence. Pour pouvoir comparer quantitativement les spectres expérimentaux aux résultats de ce modèle, il faudra également prendre en compte les états (branches) intermédiaires qui permettent des excitations multiphotoniques directes séquentielles et induisent des quasi-résonances qui augmentent considérablement les sections efficaces de transition ( resonantly enhanced multiphoton »). Du fait de la structure dense des états électroniques dans la bande de conduction du solide, ces transitions paraissent très probables et doivent contribuer à l'échauffement d'électrons vers les hautes énergies cinétiques pendant quelques dizaines de femtosecondes.

Ces premiers résultats expérimentaux et théoriques constituent une avancée importante permettant de mieux comprendre les mécanismes

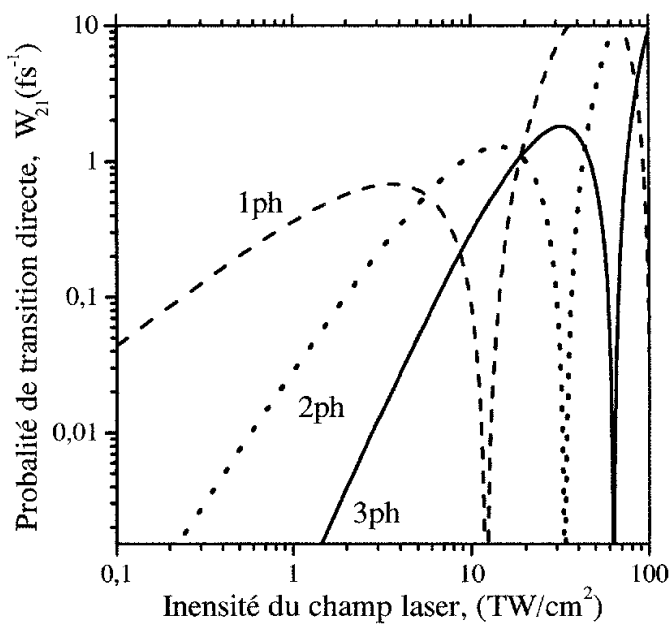

Figure 3. Probabilités/fs de transition à 1,2 et 3 photons. d'absorption d'énergie par les électrons d'un solide diélectrique soumis à une impulsion laser brève et intense et en particulier, le pré-échauffement des électrons avant la création d'un plasma et les mécanismes physiques de claquage des matériaux optiques.

\section{Références}

[1]. V. Bagnoud, F. Salin, Appl. Phys. B 70 (2000) S165-S170.

[2]. Ph. Martin, H. Merdji, S. Guizard, G. Petite, F. Quéré, B. Carré, J.F. Hergott, L. Le Déroff, P.

Salieres, O. Gobert, P. Meynadier and M. Perdrix, Laser Physics 10 (2000) 1.

[3]. G. Geoffroy, S. Guizard, E. Constant, E. Mével, A. Belsky, P. Martin, J. de Phys. IV, ce volume.

[4]. G. Petite, P. Agostini, R. Trainham, E. Mevel and P. Martin, Phys. Rev. B 45 (1992) 12210

[5]. A. Kaiser, B. Rethfeld, M. Vicanek, G. Simon, Phys. Rev. B 61 (2000) 11437.

[6]. D. Arnold, E. Cartier, and D.J. DiMaria, Phys. Rev. B 49 (1994) 10278.

[7]. B.C.Stuart, M.D. Feit, S. Herman, A.M.Rubenchik, B.W.Shore and M.D.Perry, Phys. Rev. B 53(1996) 1749.

[8]. Ph. Daguzan, S. Guizard, K. Krastev, P. Martin, and G. Petite, Phys. Rev. Lett. 77 (1994) 2352.

[9]. Vaisburd D.I, P.A.Polianov, B.N.Semin, J. Appl. Spectroscopy 62 (1995) 130.

[10]. A.N. Vasil'ev, Y. Fang, and V.V. Mikhailin, Phys. Rev. B 60 (1999) 5340.

[11]. L.V. Keldysh, Zh. Eksp. Teor. Fiz. 47(1964) 1945, Sov. Phys. JETP 20(1965).

[12]. N.M. Kroll and K.M. Watson, Phys.Rev. A, 8, 804 (1973) 